Research report

\title{
On knowing how to do things: a theory of motor imagery
}

\author{
John Annett \\ Department of Psycholoy, University of Warwick, Coventry CV4 7AL, UK \\ Accepted 22 August 1995
}

\begin{abstract}
The distinction between 'knowing how' and 'knowing that' is fundamental to current theories of cognition. Two distinct encodings or representations are implied, one conscious and verbalisable and the other normally unconscious yet demonstrable in behaviour. The paper discusses the nature of these two kinds of representation and relations between them. It is shown that imagery forms an essential mediating link between the two encodings and a theoretical model - the Action-Language-Imagination or ALI model - is presented. An important feature of the model is the role attributed to the motor system in generating imagery and principal features of motor imagery are reviewed in the context of the ALI model and with reference to recent experimental findings. Problems in mapping conscious representations of action onto physical brain mechanisms are briefly discussed. It is proposed that the physical basis of imaginal representations of actions is best understood in terms of the mechanisms of motor control. A two stage theory of motor imagery is proposed in which the first stage, the generation of a prototypical action is virtually identical to that involved in overt actions whilst the second stage depends on the retrieval of sensory impressions from memory.
\end{abstract}

Keywords: Procedural/declarative knowledge; Motor imagery; Action prototype

\section{Procedural and declarative knowledge}

The distinction between two kinds of knowledge, 'knowing how' and 'knowing that' was made nearly half a century ago by the philosopher Ryle [15]. 'Knowing that' is evidenced by a verbal statement, for example I know that "the big wheel in the Prater pleasure gardens was designed and built by British engineers", whilst 'knowing how' is evidenced by performance, actually building the wheel. Ryle was attacking the 'dualist' theory that mental events belong to a separate ghostly world of knowledge comprised of propositions which act in some mysterious way to cause overt actions. He pointed out that although we can mentally rehearse an action before, or instead of, executing it this is by no means necessary. In fact it is sometimes quite difficult to explain verbally perfectly normal routine actions. The distinction between 'procedural' and 'declarative' knowledge' and the closely related distinction between 'implicit' and 'explicit' knowledge is familiar in the writings of recent cognitive psychologists such as Anderson [1]. Both sets of terms refer to the problem of representation. Declarative or explicit knowl-

\footnotetext{
* Corresponding author. Fax: (44) (1203) 524225; E-mail: psrba@snow.csv.warwick.ac.uk
}

edge is available to consciousness, and can readily be expressed verbally, whereas procedural or implicit knowledge is typically unconscious and can only be expressed verbally with some difficulty and sometimes not at all.

Knowledge of either kind implies a representation and this symposium is about the representation of motor acts or, more generally, representations of procedural knowledge. The translation between procedural and declarative knowledge is required whenever it is necessary to explain a procedure to someone else and where a demonstration, often the preferred means of communication, is not possible the translation is often aided by the use of imagery. The procedure is first enacted in imagination and only then is available as declarative knowledge. In this paper I shall outline some ideas about imaginary actions and motor imagery from the point of view of a cognitive psychologist but also try to make some connections with neurology and physiology in terms of the kinds of physical evidence relevant to the functional analysis.

\section{Action, language and imagination}

Over a number of years I have tried to develop a framework within which to integrate ideas on the relation- 


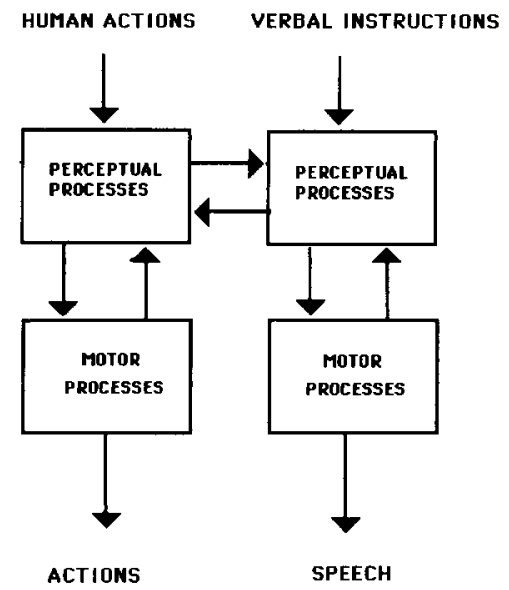

Fig. 1. The ALI model. The model represents two channels tuned to two classes of external input, human action and verbal communications. Each channel recognises and stores inputs of the appropriate type and is capable of generating corresponding outputs. The descending pathways represent simple imitation (in the case of the verbal channel reading is an example of imitation). The motor or executive precesses may be decoupled from external input in passive observation and the representational processes are decoupled from both input and output during imagery. The two channels are linked at the repesentational level by the Action-Language Bridge so that a verbal instruction to carry out an action may be transmitted from the verbal to the action channel. The bridge can also take traffic in the reverse direction as when a perceived action is described by an observer. Translation from procedural to declarative knowledge may require the subject to generate an internal representation comprising action prototypes and memories of perceptual experiences derived from previous instances of overt behaviour. This then becomes accessible to the verbal system but may also generate some motor output.

ship between declarative and procedural knowledge and the process of learning motor skills from demonstrations, verbal descriptions and instruction [2-4]. The functional model shown in Fig. 1 has four elements which are separated both vertically and horizontally and connected as shown. The vertical division distinguishes two largely independent information processing systems or channels, the action system to the left and the verbal system to the right, which each receive special classes of input, have their own storage and output facilities dealing with actions and verbal instructions, respectively.

Both action and language channels are divided horizontally with a perceptual/representational above and a motor/executive system below. The representational systems provide the means of recognising and interpreting appropriate inputs, specifically the actions of other individuals and their linguistic outputs, either spoken or written. Little needs to be said about the mass of evidence supporting the functional and physical independence of verbal and non-verbal systems. However, the case for an action system especially tuned to the encoding and interpretation of actions has not received the same degree of attention. It can be argued that an action representation system is of considerable value to a social species in interpreting the actions and intentions of other members of the group. Such a system would be on a par with the language system in facilitating communication. Whether the system is innate or acquired will not be argued here but the work of Johanssen [12] suggests it is remarkably effective without special training and that sensitivity to certain invariant features of action, for example of posture and gait, permits rapid and accurate interpretation of the identity, intentions and other physical and psychological characteristics of individuals. Just as the verbal/linguistic system can interpret a population of words and phrases so it is proposed the action system uses a 'vocabulary' of elementary actions or action prototypes. These would include elementary acts such as grasping, lifting, pointing and so on, but also non-verbal equivalents to familiar phrases or cliches. For example a squash coach I know tells his pupils "hold your racquet as if you were on the war-path waving a tomahawk". This statement encapsulates a complex behaviour pattern which is readily understood and reproduced. In Fig. 1 the connection between verbal and action representation at the level of action prototypes is direct and effective. Sports coaches do not, in general, give verbal instruction in terms of the detailed kinematic features of the actions they are trying to train.

The action representation system involves both conscious and unconscious processes. The visuo-spatial sketchpad (see Logie, this issue) provides a conscious arena for perceiving or imagining actions, whilst action prototypes are presumed to be held in an unconscious long term memory but enter consciousness when activated. It is further hypothesised that there is a close interaction between the representation and the executive systems. Specifically, when an action prototype is activated it will provide control signals for the executive system. Both motor empathy and motor imagery would seem to require such a mechanism. However, the representative and executive systems can be decoupled such that perception is not inevitably translated into imitative action and that actions can be imagined without being translated into overt movement. Both perception and imagery must therefore involve a very precisely tuned inhibitory mechanism. Just as young children must learn to read silently after first having learned to read aloud, so also they tend to imitate their parents and older siblings before gaining full independent control of their own actions.

This model has the following implications:

1. It provides a framework for understanding imitation by means of the action prototypes which can be observed, stored and reproduced.

2. It provides a framework for understanding motor imagery since activation of a prototype may bring certain features of the action into consciousness without necessarily involving muscular activity.

3. It provides a framework for understanding the relationship between procedural and declarative knowledge. To explain how to do something the relevant prototypes are activated and, via conscious images, enable the verbal system to make appropriate statements. Connectivity 
between the action and language systems at the representational level also permits verbal instruction to be translated into action. This two-way traffic is symbolised by the metaphor of an action-language bridge.

\section{A theory of motor imagery}

Theories of motor imagery fall into one of two main categories. The first emphasises the 'symbolic' content of the imagery, motor imagery is said to comprise the activation of a central action plan. The second emphasises the continuity of overt and covert action, the latter being simply a scaled down version of the former and which includes motor output and some kinesthetic sensation. Jeannerod [11] has recently proposed a theory of the former kind, namely that motor imagery constitutes the preparation for action. The theory I propose has elements of both these viewpoints. First, we go back to William James's notion of the 'sense of effort' [10]. This is the feeling, or rather the knowledge, that one is the agent of perceptual changes. Even the weakest forms of imagery contain this subjective element and this is what distinguishes imagery from hallucination. In addition subjects report experiences resembling those which would be expected whilst performing the overt action and these have the characteristics of memory recall. The proposed theory is that motor imagery contains these two elements, first the activation of the action prototype and second, memories of the perceptual consequences of previous actions of a similar kind. The first element is shared with overt action whilst the second is a substitute for the sensory feedback which would normally arise from the overt action.

An action such as manipulating an object involves the activation of the relevant action prototype, for example the type of hand grip afforded by the object. Action prototypes are modified by the addition of location and timing information but in imaginary movement the value of these parameters is modified such that overt movement is minimised. According to Woodworth's classical theory of motor control [17] this initial specification determines the course of the action for the first $200-300 \mathrm{~ms}$ after which internal and external feedback would provide further inputs which can be used to modify the further course of the action. The delay or absence of normal feedback can disrupt control of all but short duration actions but in imaginary action substitute feedback is available from memory.

These two elements of the theory are illustrated by an analysis of behaviour and subjective reports in a test in which the subjects is asked to describe how to tie a bow [3] without physically performing the actions. Verbal reports typically refer to a series of elementary steps in which one act, say picking up the string, has to be completed before the next step, crossing the strings, can begin. Subjects often report that the images are experienced as a series of such steps, like photographic stills each being both the product of the preceding intention and the "stimulus' to the next and I have yet to meet a subject who claims to be able to complete the task without the use of imagery. Quite often the initial act is repeated, apparently as an aid to generating a picture of its completion, but as soon as this is done the next action prototype, say forming a loop, is activated, and so the series of elementary acts which make up the whole task is completed. Sometimes an elementary act is omitted from the verbal account but acts never occur in the wrong order. The analysis of partially suppressed hand gestures is entirely consistent with the notion that motor imagery involves the activation of prototypes decoupled from the parameters which would have to be fed in to control actual movement. They tend to convey the form of the action but use arbitrary distance and direction information. Whilst the fingers are seen to be grasping an imaginary object this is located in a different part of space and actions, such as pulling the string to tighten the knot, are typically larger than would actually be required. The action may even be expressed through a totally different set of muscles, for instance a subject may indicate the shape of the movement required to make a loop by gesturing with the head, using the nose to indicate the shape. The reported imagery is often highly detailed suggesting it is based on specific memories. One subject explained bow tying by recalling tying training shoes, even recalling the characteristic smell of the school gymnasium, and where subjects have recently been required to tie a bow they tend to recall details, such as the colour of the string used, derived from that particular instance.

\section{The search for the physical bases of motor imagery}

The notion of 'functional equivalence' is central to imagery research. Finke [8] suggested that visual images share at least some of the brain processes involved in 'normal' perception and so we naturally ask whether and to what extent motor imagery shares the physical mechanisms of overt action - put simply, where in the brain is motor imagery located?

The first point to make is that the influence of Bernstein [6] has moved motor theorists away from the notion that movement is dependent on stored motor programmes both on the grounds that actions are so varied that the storage of complete and detailed motor programmes is almost inconceivable and that observed movement is due to a subtle interplay between neural mechanisms and the physical forces acting on the body. Walking, for example, can be described as a controlled falling forward in which small changes in posture enable gravity to pull the body forward whilst precisely timed leg movements limit the fall. Even the leg movements themselves are determined in different phases by a combination of voluntary muscle contraction and the action of gravity of the mass of the limb. The still 
open question is how much, or how little, information needs to be stored for the execution of learned actions and this obviously has important implications for the question of the functional equivalence of motor imagery and overt movement.

The theory I have just outlined implies two distinct storage systems, one a truly 'motor' store which retains a number of basic action prototypes which can be activated by associative processes, and the other comprising recollections of the sensory experiences associated with past actions. The former are employed equally in both imaginary and overt movement whilst the latter are only invoked when 'normal' feedback is unavailable due to the decoupling of the activated prototype from the executive system. The theory thus implies that 'functional equivalence' is complete for the former but may be only partial as regards the latter component.

I will consider briefly some of the neurological and physiological evidence which has a bearing on this issue without any pretence of providing a complete account. The recent report of di Pellegrino et al. [7] provides evidence of a specific neural mechanism capable of storing action prototypes, moreover the evidence matches exactly the requirement of my general model that there should be a mechanism which is active in both the production and the recognition of specific actions. Using single cell recording in the rostral part of the pre-motor cortex of Macaca nemestrina certain cells were found which responded only to specific meaningful patterns of action and did so whether the action was self-performed or whether the monkey simply observed the experimenter performing the same action.

R. Smith and I [5] have obtained evidence of a different kind which has a bearing on the decoupling of the action schema from the executive system. The performance of a group of 14 patients with moderately severe Parkinson's disease was compared with that of normal controls, usually the patient's spouse, on the bow tying task described above. The PD patients when asked to tie a bow managed to do so but took on average three times as long as the normal controls. However, when asked to explain how to tie a bow the same patients performed just as well, in fact slightly better than their normal controls both in the quality of their verbal reports and the time taken to make them. The conclusion must be that, for this task at least, imagery is functionally normal; disease of the basal ganglia does not interfere with either the activation of action prototypes or the retrieval of memory images. In overt action the problem for PD patients is often an inability to relate the magnitude of the required movement to the physical parameters of the current situation but this is precisely what is not needed in the case of imaginary actions.

The third piece of evidence is extremely tentative since it relates to an as yet unfinished experiment. If the theory of motor imagery has any merit then we should look carefully at neural events near the beginning of the imagi- nary actions. The methods of rCBF and SPECT scanning classically used by Roland et al. [14] and by Goldenberg et al. [9] have relatively good spatial resolution but rather poor temporal resolution. At Warwick we have recently begun some experiments using BEAM which has a much better temporal resolution (about $2.5 \mathrm{~s}$ ) but relatively crude spatial resolution [16]. One experiment using event related potentials (ERP) provides information over the first second following an event. Subjects make a simple unimanual linear movement of about $30 \mathrm{~cm}$ from left to right at the onset of a tone whilst EEG records are taken. Another set of records is then taken with the subject instructed simply to imagine making the movement. The waveforms for 40 trials are summed and show a typical pattern of a negativity at around $200 \mathrm{~ms}(\mathrm{~N} 200)$ following the stimulus and a positivity at around $300 \mathrm{~ms}(\mathrm{P} 300)$. Bearing in mind that the theory requires that neural events up to $200-300 \mathrm{~ms}$ should be essentially the same for actual and imagined movement we were interested to find highly significant differences between these conditions beginning to emerge at around 300 msecs. Picton [13] suggests that P300 is associated with conscious information processing. During actual movement the P300 amplitude is significantly greater over the frontal areas than in either control or imagery conditions, however under the imagery condition frontal activity is no different from that under control conditions but amplitude in the right parietal area is significantly increased. Whilst I should emphasise these are only very preliminary results from a group of 11 subjects, they appear to fit closely to the expectation that whilst initially overt and imaginary movement are indistinguishable on the EEG record, shortly after the initiation of the imaginary movement the cortical area concerned with spatial perception is relatively more active than the primary motor areas.

\section{Summary}

Procedural knowledge, or 'knowing how to do things', is typically unconscious but can be translated into verbally accessible declarative knowledge by self-observation during physical or imaginal enactment. A framework for understanding this process also provides some insights into learning by imitation and verbal instruction and the nature of motor imagery.

A 'channel' specialised in the recognition, storage and production of actions is proposed and this includes a representational sub-system and an executive sub-system. The representational sub-system includes both conscious and unconscious components, the latter including stored schematic or prototypical actions. The two sub-systems are closely coupled in action production but can be decoupled during perception and imagery.

A two-stage theory of motor imagery is proposed. In the first stage an action prototype is activated whilst the 
executive sub-system is decoupled and in the second phase, beginning $200-300 \mathrm{~ms}$ later, imaginal sensory feedback is generated by accessing memories of previous enactments.

There is evidence from single cell recordings of a possible physical basis for action prototypes which have both perceptual and executive functions. Some recent results from neuropsychological and psychophysiological studies are consistent with the proposed decoupling of representational and executive functions in motor imagery and the separate activation of action prototypes and sensory memory during imagined actions.

\section{References}

[1] Anderson, J.R., Cognitive Psychology and its Implications, W.H. Freeman, New York, 1980.

[2] Annett, J., Action, language and imagination. In L. Wankel and R.B. Wilberg (Eds.), Psychology of Sport and Motor Behavior, University of Alberta, Edmonton, 1982, pp. 271-281.

[3] Annett, J., On knowing how to do things. In H. Heuer and C. Fromm (Eds.), Generation and Modulation of Action Patterns, Springer, Berlin, 1986, pp. 187-200.

[4] Annett, J., Relations between verbal and gestural explanations. In G.E. Hammond (Ed.), Cerebral Control of Speech and Limb Movements, North Holland, Amsterdam, 1990, pp. 327-346.

[5] Annett, J. and Smith, R., Motor imagery in Parkinson's disease. In
C. Cornoldi (Ed.), Pre-proceedings of the Second International Workshop on Imagery and Cognition, Padua, 1988, pp. 373-388

[6] Bernstein, N., The Co-ordination and Regulation of Movements, Pergamon Press, Oxford, 1967.

[7] Di Pellegrino, G., Fadiga, L., Fogassi, L., Gallese, V. and Rizzolati, G., Understanding motor events: a neurophysiological study, Exp. Brain Res., 19 (1992) 176-180.

[8] Finke, R.A., The functional equivalence of mental images and errors of movement, Cogn. Psychol., 11 (1979) 235-264.

[9] Goldenberg, G., Podreka, I., Steiner, M., Willmes, K., Scuss, E. and Deeke, L., Regional cerebral blood flow patterns in visual imagery, Neuropsychologia, 27 (1989) 641-664.

[10] James, W., Textbook of Psychology, McMillan \& Co., London, 1892.

[11] Jeannerod, M., The representing brain: neural correlates of motor intention and imagery, Behav. Brain Sci., 17 (1994) 187-245.

[12] Johansson, G., Visual motion perception, Sci. Am., 323 (1975) $76-88$.

[13] Picton, T.W., The P300 wave of the human event-related potential, J. Clin. Neurophysiol., 9 (1992) 456-479.

[14] Roland, P.E., Larsen, B., Lassen, N.A. and Skinhoj, E., Supplementary motor area and other cortical areas in organisation of voluntary movements in man, J. Neurophysiol., 43 (1980) 118-136.

[15] Ryle, G., The Concept of Mind, Hutchinson, London, 1949.

[16] Williams, J.D., Rippon, G. Stone, B.M. and Annett, J., Psychophysiological correlates of dynamic imagery, Br. J. Psychol., 86 (1995) 283-300.

[17] Woodworth, R.S., The accuracy of voluntary movement, Psychol. Rev. Monograph Suppl., 3, No.3. 